\title{
The Spitzoid lesion: rethinking Spitz tumors, atypical variants, 'Spitzoid melanoma' and risk assessment
}

\author{
Raymond L Barnhill \\ Departments of Dermatology and Pathology, University of Miami Miller School of Medicine, Miami, FL, USA
}

\begin{abstract}
Although much remains to be learned about Spitzoid lesions, there is increasing evidence that these tumors may be a type of melanocytic neoplasm distinct from conventional melanocytic nevi and malignant melanoma. In the current communication, the author has attempted to describe accurately the state-of-the-art surrounding these lesions, their nomenclature, and assessment of risk. Acknowledging the peculiar nature of Spitzoid lesions, the author prefers the term Spitz tumor rather than 'Spitz nevus' (except perhaps for the most typical lesions) and argues against using the term 'Spitzoid melanoma' until more information is available to justify such a term. The author also believes that patients are best served by the comprehensive evaluation of Spitzoid lesions and their classification into three categories: (1) Spitz tumor without significant abnormality, (2) Spitz tumor with one or more atypical features (atypical Spitz tumor), including those judged to have indeterminate biological potential, and (3) malignant melanoma, rather than the two categories of 'Spitz nevus' and melanoma. Only rigorous characterization of sufficient numbers of Spitzoid lesions and long-term follow-up of patients will provide truly objective information for the formulation of optimal guidelines for the management of patients with these lesions.
\end{abstract}

Modern Pathology (2006) 19, S21-S33. doi:10.1038/modpathol.3800519

Keywords: Spitz nevus, Spitz tumor, melanoma

In 1910, Darier and Civatte described in some detail an unusual (Spitzoid) melanocytic tumor developing rapidly on the nose of a young child, and they were completely thwarted in their efforts to decipher whether the lesion was benign or malignant. ${ }^{1}$ One century later, one can question whether pathologists have made any real progress in resolving this very same conundrum: the inability to accurately interpret many such Spitzoid lesions (see Note) histologically and to know their biological potential! Of course, over these ensuing 95 years a certain amount of information has been collected about these lesions and guidelines have been proposed for distinguishing 'Spitz nevi' from malignant melanoma (Tables 1 and 2). ${ }^{2-24}$ Because of these guidelines for diagnosis, many dermatopathologists may, on the surface, appear to be at ease or to have greater confidence in dealing with this problem. However, the reality of the situation is that a large

Correspondence: Dr RL Barnhill, MD, Departments of Dermatology and Pathology, University of Miami Miller School of Medicine, and Global Pathology Laboratory Services, 16250 N.W. 59th Avenue, Suite 201, Miami Lakes, FL 33014, USA.

E-mail: rbarnhill@mad.miami.edu

Received and accepted 7 October 2005 proportion of these lesions remain a daunting challenge to histopathological interpretation and the diagnosis rendered is in many instances tentative, probabilistic, and often arbitrary.

The aim of this paper is not to review exhaustively the evolution of knowledge and thinking about Spitzoid lesions over the past century but rather to make certain observations and to offer a philosophically practical approach to the difficult Spitzoid tumor.

It is useful to revisit the historic paper published by Sophie Spitz ${ }^{2}$ in 1948 . Her article was entitled 'Melanomas of childhood' indicating that Spitz did not consider these lesions as a group to be 'unequivocally benign nev(i) of childhood' nor malignant melanomas of adults. Spitz also concluded that 'differentiation histologically between the juvenile and adult melanomas could not be made with certainty in most cases', and this was evidenced by fatal metastases developing in one of 13 cases reported by Spitz. The latter point is reinforced by recent experience. In 1999, we described one case (among thirty lesions studied) thought by consensus to be a 'typical Spitz nevus' that ultimately resulted in death of the patient. ${ }^{25}$ Furthermore, Spitz thought that the onset of puberty was a singularly important 
factor in the rise in mortality from melanoma noted with age and the extreme rarity of death from melanocytic lesions before puberty. It is noteworthy

Table 1 Characteristics of conventional Spitz tumors

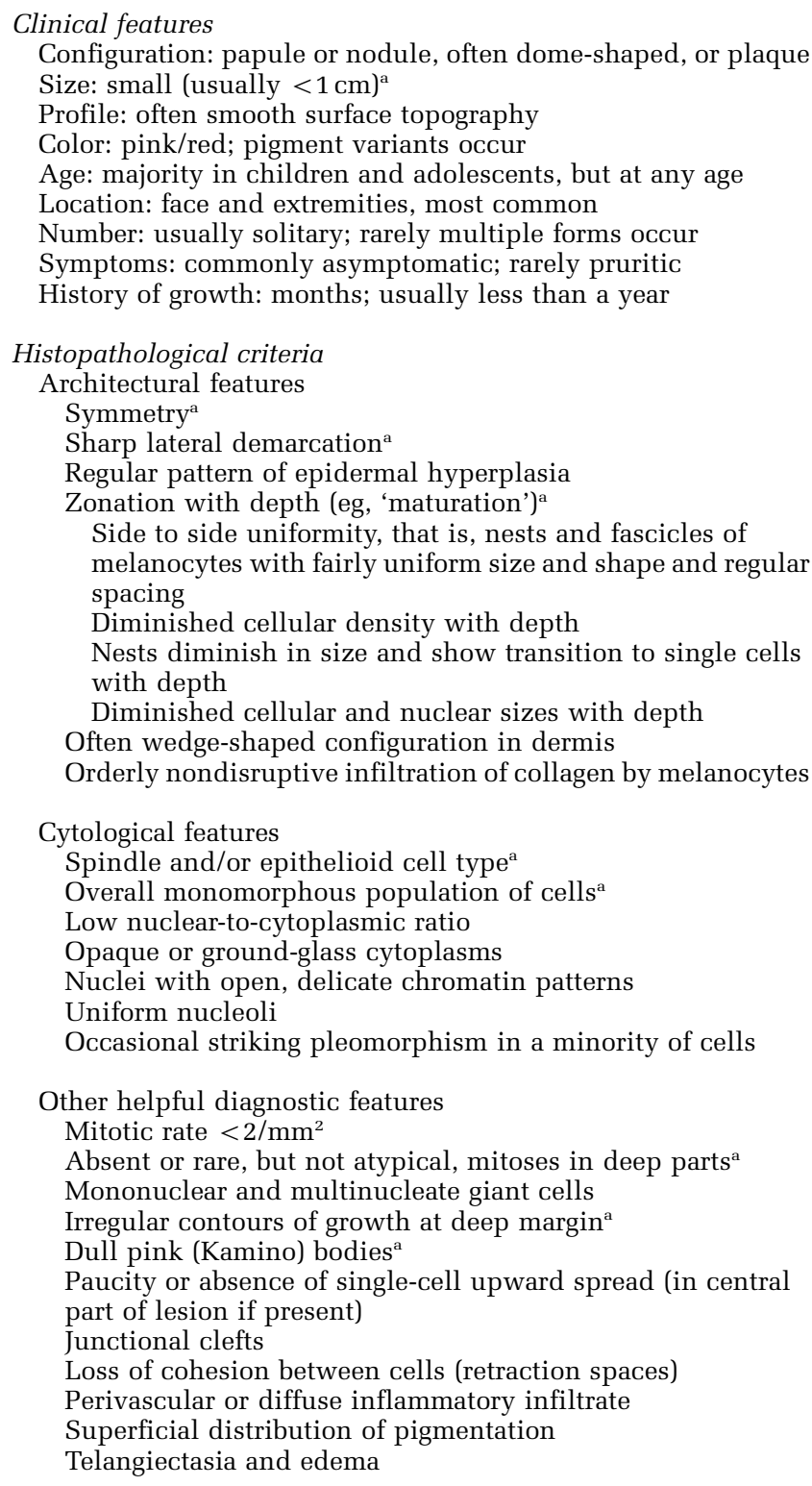

${ }^{\mathrm{a}}$ Most helpful features. that both Spitz's and our fatal cases developed in 12year-old female subjects who undoubtedly were in puberty or were postpubertal. One is left with the distinct impression that Sophie Spitz remained unsettled by this group of lesions and did not at all consider them a variant of ordinary nevus.

Over the past half century there has been the progressive trend to consider the Spitz tumor as simply a variant of benign melanocytic nevi quite distinct from melanoma and to utilize the terms 'Spitz nevus, Spitz's nevus, or spindle and epithelioid cell nevus' to describe this lesion. ${ }^{3-24}$ This approach has come about from the reports of series of Spitzoid lesions and the promulgation of histological criteria from these series. As we have described various authors have reviewed their own series of 'Spitz nevi' and accordingly formulated their 'criteria' from these self-selected (and consequently biased) series of lesions (Table 2)..$^{7,11,12,26}$ The inherent flaws resulting from this exercise involve circular reasoning, the cases in general have not been population-based, the number of cases has often not been adequate, and the cases have not had long-term follow-up (of 8-10 years) to know the outcomes. ${ }^{22,25,26}$ Following up on the observations that the vast majority of these Spitzoid lesions do not seem to recur or to metastasize, they have been lumped as a group in benign melanocytic nevi, even though some proportion of lesions seem impossible to distinguish from melanoma and uncommon or rare lesions behave aggressively. Thus, the term 'Spitz nevus' has become firmly entrenched in the medical literature. The author acknowledges without question that the most typical or banal Spitz tumors share particular features with conventional acquired melanocytic nevi. One can argue that this group of Spitz lesions (that do not appreciably deviate from this characterization of a benign melanocytic lesion) should be termed Spitz nevi. However, the problem with the indiscriminate use of 'nevus' is that it connotes a lesion that is completely benign and a priori presents no risk to the patient. Obviously, this is not the case for some subset of Spitzoid lesions as has clearly been shown by Sophie Spitz herself (and many others)!

It is neither rational nor practical and it is potentially harmful to patients and physicians alike (see below) to label prospectively virtually all

Table 2 Contrasting criteria for Spitz tumors from reports in the literature ${ }^{30}$

\begin{tabular}{|c|c|c|c|c|}
\hline Criterion & Kernen and Ackerman ${ }^{7}$ & Reed et $\mathrm{al}^{26}$ & Weedon and Little ${ }^{12}$ & Paniago-Pereira et al ${ }^{11}$ \\
\hline Pagetoid spread & Not stated & Not stated & Occasional & Sparse \\
\hline Nuclear atypia & Absent & Minimal & Present & Not important \\
\hline Giant cells & Occasional & Present & Present & Not helpful \\
\hline Epidermis & Thinned & Hyperplastic & Hyperplastic & Hyperplastic \\
\hline Cell type & Mostly spindled & No comment & Mostly spindled & Mostly spindled \\
\hline Dermal mitoses & Rare & Variable (rarely high) & Common & Occasional \\
\hline Atypical mitoses & Rare & Not stated & Uncommon & Rare \\
\hline Maturation with descent & Mostly absent & Present & Mostly absent & Prominent \\
\hline Deep dermal margin & Pushing & Infiltrative & Infiltrative & Pushing \\
\hline
\end{tabular}


Spitzoid lesions as nevi and then to have to redress the issue at some later date. This usually involves reclassifying some lesions as malignant or melanoma some time later after the development of metastases or death. It is obvious that this practice also leads to the self-correcting behavior of lowering the threshold for diagnosing melanoma in order to avoid a disastrous outcome of missing melanomas and metastasizing lesions. For the latter reasons, the author favors the term 'Spitz or Spitz-like tumor' (equally acceptable terms might include Spitz, Spitz-like, or Spitzoid lesion, melanocytoma or neoplasm) and the use of an intermediate or third category lesions that are difficult to classify as clearly benign or malignant, that is, Spitz tumors that are 'atypical, controversial, or biologically indeterminate' (see below). ${ }^{27}$

It is apparent that one message stemming from the published work on Spitzoid lesions is that if one utilizes the 'established criteria' one can very easily interpret a Spitzoid neoplasm as either: (1) Spitz's nevus or (2) malignant melanoma. ${ }^{28}$ Another trend has been to interpret almost all Spitzoid lesions in children as benign 'Spitz nevi' and many Spitzoid lesions in adults (particularly beyond the age of 3040 years) as malignant melanoma, almost without any attention to the histopathological features (RL Barnhill, 2005, personal observations) As mentioned above, a corollary of the latter practice, a literal 'dumbing-down of criteria', has been to designate virtually all Spitzoid lesions as melanoma. While medical-legal concerns have understandably influenced the behavior of pathologists in this area, the latter practices often offer pathologists no better solution than that of 'flipping a coin', or literally abandoning the practice of histopathology.

The author does not believe that this overly simplistic attempt to assign an often arbitrary diagnosis of either 'Spitz's nevus' or malignant melanoma to every Spitzoid lesion ${ }^{28}$ serves patients well nor provides a realistic methodology for dealing prospectively with an often profoundly difficult problem. Such an exercise undoubtedly results in both the overdiagnosis and underdiagnosis of melanoma in a certain number of cases. Patients may unfairly suffer the psychological burden of a grave diagnosis and may undergo overly aggressive and potentially harmful therapies. At the same time, some proportion of patients will have the false and unjustified assurances of a benign diagnosis and may not receive appropriate treatment and followup. Rather, we must be honest with patients and above all try to do no harm. We must accept that currently we have a rather poor understanding of the biological nature of Spitzoid lesions and that current criteria for their diagnosis are wanting. We must adopt a pragmatic approach that attempts to avoid both the overdiagnosis of melanoma and inappropriate management of patients. Einstein's philosophy that one should try to 'make things simpler but not too simple' is especially applicable here. Thus, the
Table 3 Protocol for Spitz tumors

1. Examination of the entire lesion

2. Application of all histopathological, clinical, and other attributes for assessing abnormalities present

3. Seek consultation

4. Placement into risk category (Table 4)

5. Management of patient

Table 4 Categorization of Spitzoid lesions according to risk stratification

Spitz tumor without atypicality

Atypical Spitz tumor (Spitz tumor with one or more atypical features)

Lesions with indeterminate biological potential (lesions difficult to classify as unquivocably benign or malignant) Malignant melanoma

rather arbitrary interpretation of all Spitzoid lesions as either 'Spitz's nevus' or malignant melanoma is too simple, not realistic, and may be harmful to patients.

As the author has previously suggested, a more realistic solution is to employ a well-defined protocol (Table 3) for the systematic and rigorous evaluation of Spitzoid lesions utilizing all histopathological, clinical, and ancillary information available. ${ }^{25,27,29}$ Having collected this information, one can then assign a given lesion to one of three categories (Table 4): (1) Spitz tumors without appreciable abnormality (Figure 1), (2) Spitz tumors with one or more atypical features (atypical Spitz tumor) (Figure 2) including those with indeterminate biological or malignant potential (Figure 3), and (3) malignant melanoma. In effect, this involves risk stratification of lesions, open and honest communication with patients, and rational decision-making for patient management, that is neither harmful nor negligent. Given the imperfect state of current information, the author acknowledges that this exercise still remains largely subjective and is dependent on the knowledge, experience, and common sense of the pathologist and other physicians involved in the care of the patient.

The remainder of the paper will elaborate on this process of evaluating Spitz tumors with particular emphasis on the characteristics of atypical Spitz tumors. Finally, the controversial group of melanomas closely resembling Spitz tumors, or 'Spitzoid' melanomas, will be discussed and whether their distinction from atypical Spitz tumors is possible at present.

\section{Spitz tumors with atypical features}

The obvious goal of developing criteria for any tumor system is to be able to discriminate lesions 

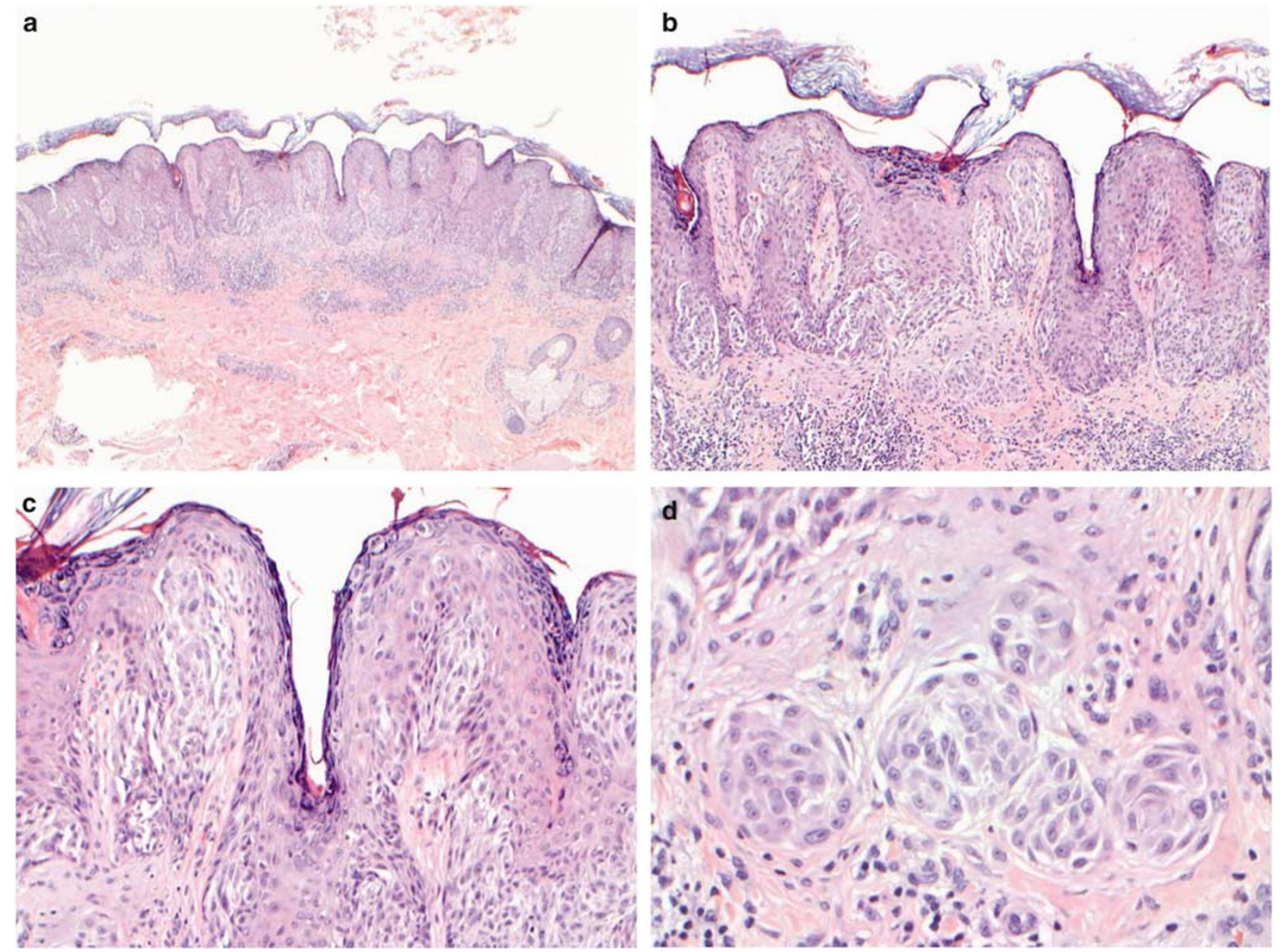

Figure 1 Compound Spitz tumor without significant atypicality of a 22-year-old male with lesion involving the left upper arm. The lesion measures less than $10 \mathrm{~mm}$ in diameter and $0.61 \mathrm{~mm}$ in Breslow thickness. Other attributes: no asymmetry, sharp circumscription, no ulceration, only minimal focal pagetoid spread, and there are no mitoses in the small dermal component. This lesion requires complete excision and the patient follow-up examinations at least once a year for example. (a) Scanning magnification show a slightly raised tumor with general symmetry. (b) The lesion demonstrates orderly appearance with regular junctional nesting and small dermal component. (c) The lesion shows focal pagetoid spread and fairly regular junctional nesting of melanocytes. (d) Note uniformity of spindle and epithelioid cells in the dermis.

that are 'malignant, behave aggressively, or have other adverse prognostic properties' from those that are biologically 'benign' or pose no threat to the patient. We set out to test the hypothesis as to whether the generally accepted 'criteria' for Spitz nevi truly discriminate 'benign' lesions from aggressive or malignant lesions in a paper published in $1999 .{ }^{25}$ The latter study involved a blinded review of 30 melanocytic lesions exhibiting features of Spitz nevus (the cases included 'typical Spitz nevi', Spitz tumors with atypical features, biologically indeterminate Spitz-like lesions, and unequivocal melanomas) by 10 pathologists. The outcome of the project showed that this panel of pathologists could not reach consensus about diagnosing such Spitz lesions or distinguishing them from melanoma using the conventional criteria from the literature. ${ }^{25}$ As has already been mentioned one of the cases thought by consensus to be a typical 'Spitz nevus' eventually resulted in death of the patient. Therefore, we concluded that even among experts the published guidelines for the dichotomous distinction of Spitz nevi from melanoma were inadequate. The latter work has been the incentive for the ongoing study and formulation of criteria for and risk stratification of Spitzoid lesions (Tables 5 and 6). ${ }^{30}$

\section{Background of the atypical Spitz tumor}

The term 'atypical Spitz tumor' was first used in the English language literature (to the author's knowledge) in 1975 by Reed et $a l^{26}$ in illustrating a Spitz tumor (in a photomicrograph) that differed from conventional Spitz nevi by exhibiting confluent and densely cellular fascicles of spindle cells that 'crowded and compressed their stroma'. In a subsequent report of 32 cases, Smith et al $3^{31}$ introduced the term 'spindle and epithelioid cell nevus with atypia and metastasis (malignant 

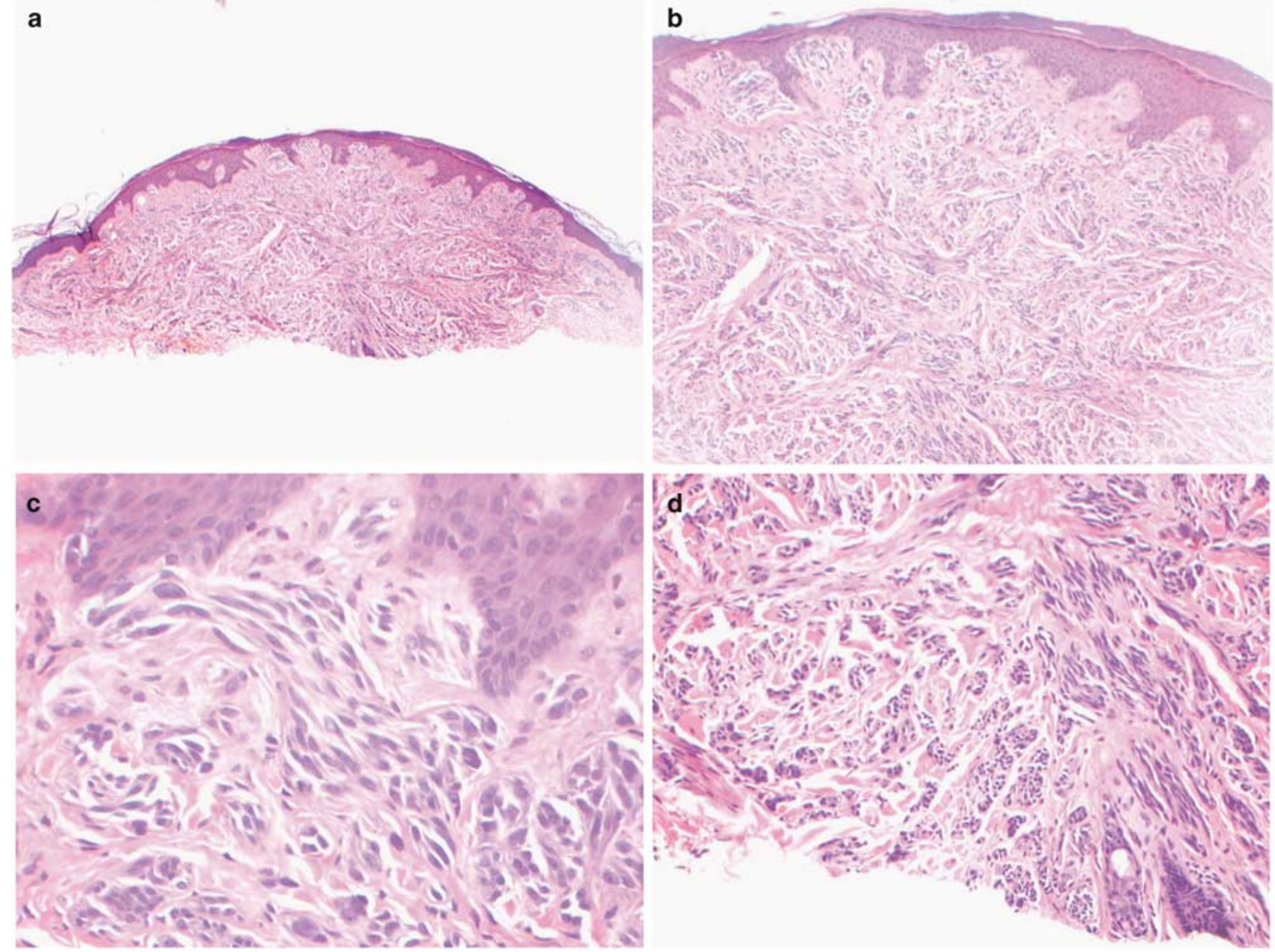

Figure 2 Compound Spitz tumor with atypical features of a 36-year-old woman with lesion from the right thigh. The tumor measures less than $10 \mathrm{~mm}$ in diameter and at least $2.1 \mathrm{~mm}$ in Breslow thickness. Other attributes: slight asymmetry, reasonable circumscription, no ulceration, no pagetoid melanocytosis, lack of maturation, high cellular density and confluence of melanocytes in dermis, $2 / \mathrm{mm}^{2}$ mitotic rate, deep mitosis, and prominent nuclear pleomorphism. This lesion lacks sufficient atypicality in the author's experience for conventional melanoma. Such a lesion requires re-excision with margins of about $1 \mathrm{~cm}$ and careful follow-up at least every 6 months. SLN biopsy may be considered at some institutions but has no proven benefit. (a) Scanning magnification shows a raised dome-shaped tumor with the general appearance of Spitz tumor and slight asymmetry. (b) Note lack of maturation of dermal component. (c) The lesion exhibits junctional nesting of melanocytes but no pagetoid spread. The nests of melanocytes display prominent cellularity and confluence. (d) There is no maturation at the base of the tumor.

Spitz nevus)' (see below) to describe a series of Spitz-like melanocytic tumors characterized by large size $(>1 \mathrm{~cm})$, frequent ulceration, deep extension into subcutaneous fat with bulbous 'pushing' margins, prominent cellular density, lack of maturation, cytological atypia greater than that expected for a 'Spitz nevus' (large and pleomorphic cells, prominent nucleoli), significant numbers of mitoses (up to 5 in a single high-power field), and focal necrosis. These tumors developed in relatively young individuals $(41 \%<14$ years and $82 \%<29$ years of age), and virtually all were located on the head and neck, and extremities (71\%). Six patients were also observed to have regional lymph node metastases with involvement of the sinuses and parenchyma by tumor identical to the primary cutaneous lesion. According to the authors, there was no subsequent progression of the disease; however, long-term follow-up of these patients has not been reported. There were no distinctive features that predicted the development of metastases.

The author has reported a series of 12 atypical Spitz-like melanocytic tumors in children and adolescents. ${ }^{29}$ There were three tumors (classified as melanoma) among the 12 cases that were associated with metastases and the death of one patient. Two of the latter patients had only single lymph node metastases and have been disease-free with long-term follow-up. The latter two tumors also potentially could be described as 'metastasizing Spitz tumors'. In general, all 12 tumors were characterized by many of the same features as reported by Smith et $a l^{31}$ that is, large size, ulceration, significant depth, prominent cellular density, lack of maturation, a pushing border, 
a
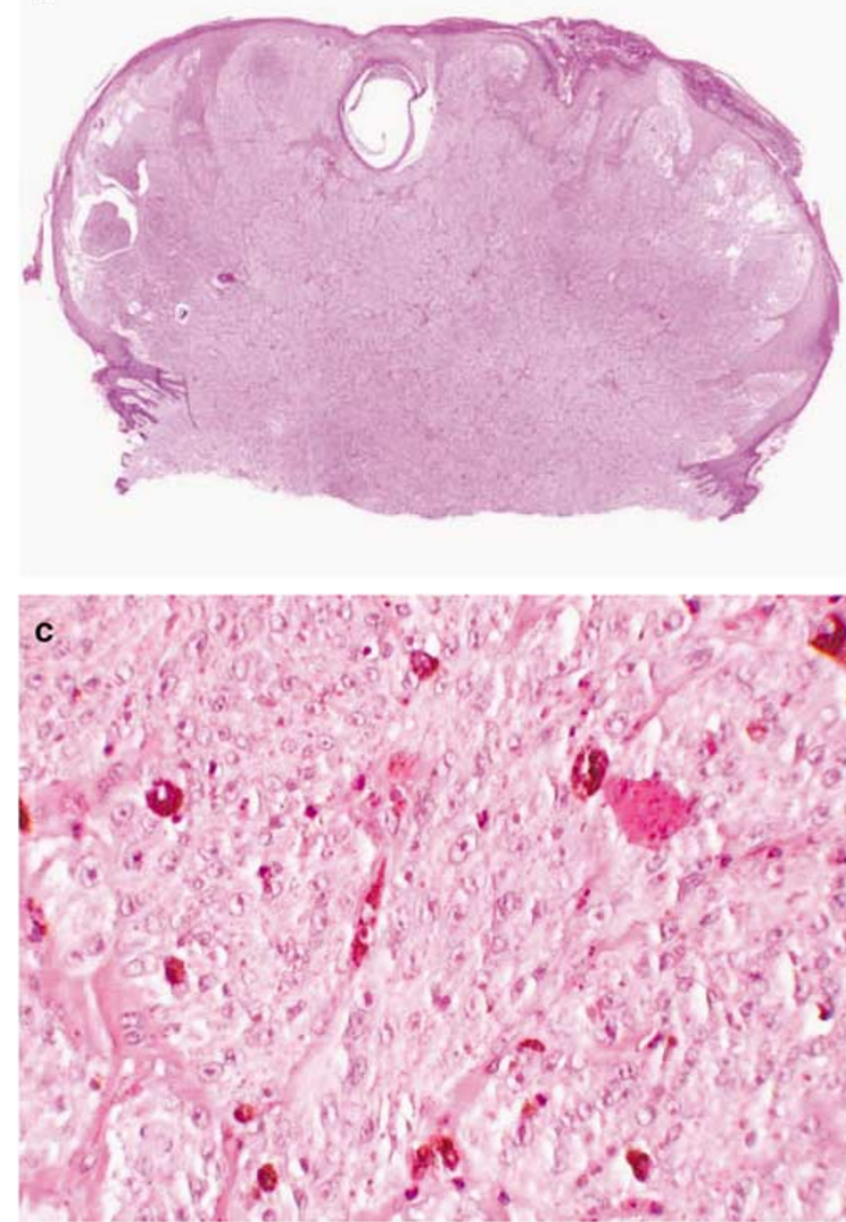
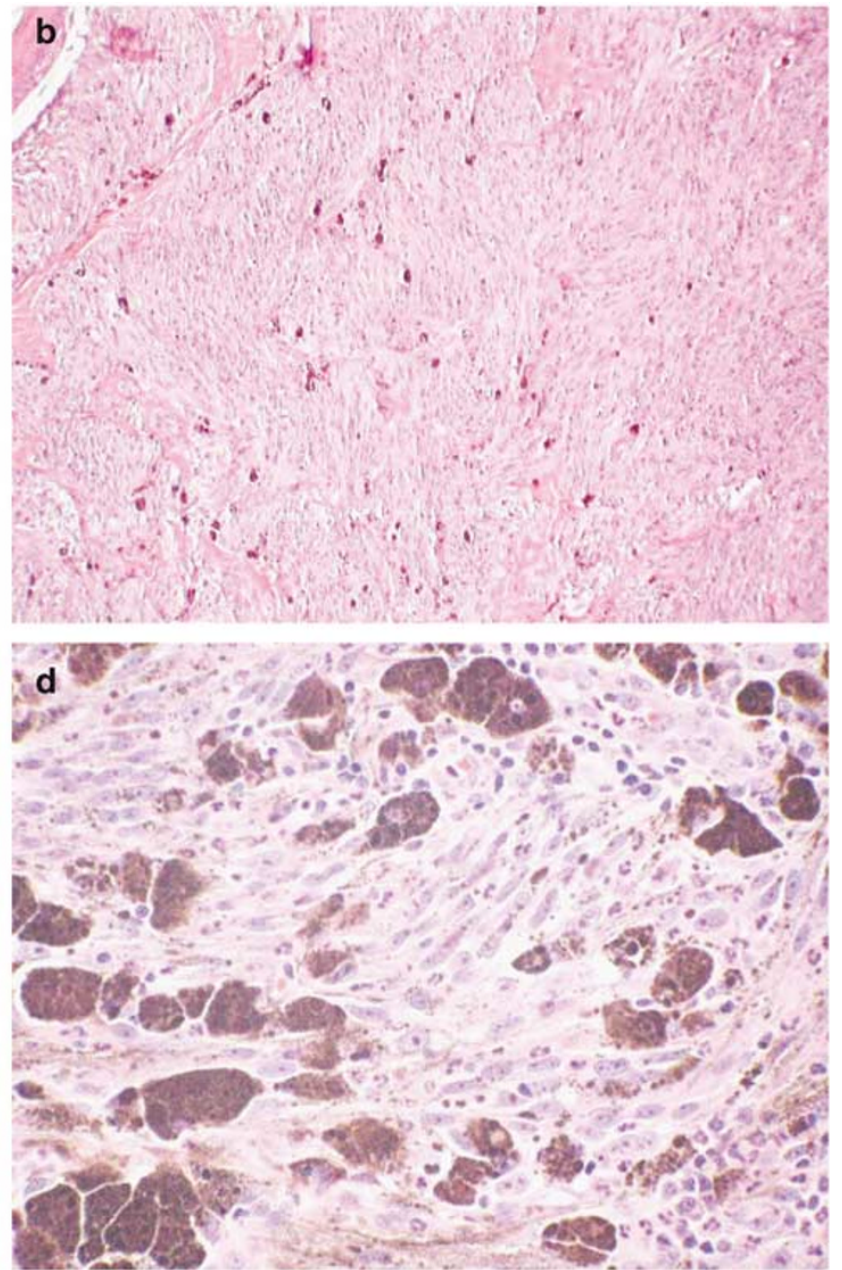

Figure 3 Malignant melanoma (or metastasizing Spitz tumor?) of a 7-year-old boy with lesion from the left back initially diagnosed as atypical Spitz nevus at an another institution. The tumor measures $9 \mathrm{~mm}$ in diameter and at least $4.4 \mathrm{~mm}$ in Breslow thickness. Other attributes: asymmetry, reasonable circumscription, no ulceration, no pagetoid melanocytosis, no maturation, high cellular density and confluence of melanocytes throughout dermis, $9 / \mathrm{mm}^{2}$ mitotic rate, deep mitoses, and prominent cytological atypia. The lesion recurred in a matter of months and two SLNs contained large deposits of an atypical melanocytic tumor. Long-term follow-up will be needed to determine whether there is disease progression or not. (a) Scanning magnification shows a large raised polypoid tumor with slight asymmetry. (b) The superficial portion of the tumor is characterized by confluent sheets of melanocytes replacing dermis. (c) Note striking density of melanocytes at base with cytological atypia. Many nuclei contain prominent nucleoli. (d) SLN containing large tumoral deposit replacing large part of node. Note pronounced cytological atypia of spindled melanocytes.

variable but often frequent and deep mitosis, and prominent cellular pleomorphism.

From the reports cited above, it is apparent that there is a not only an experiential but also a factual basis for formulating criteria for Spitzoid lesions with abnormal features and that these attributes potentially may have predictive value for risk stratification (and the rational for an intermediate category of Spitzoid lesions) (Table 5). ${ }^{29}$ Based on the information from the cases referred to we have developed the grading protocol outlined in Table 6 to aid in the assessment of metastatic risk of Spitz tumors with abnormal features in children and adolescents. $^{30}$ In an evaluation of 30 atypical Spitz-like melanocytic tumors, an increasing score resulted in greater risk for the development of metastases.

\section{Criteria for the assessment of Spitz tumors}

A Spitzoid lesion should be initially screened histopathologically to determine whether an obvious melanoma is present. If clearcut melanoma can be excluded, Spitz-like tumors should be systematically assessed with the following histopathological, clinical, and ancillary parameters ${ }^{30}$ (Table 5) (Figures 1-3).

\section{Histopathology}

\section{Organizational attributes}

Size (diameter in $\mathrm{mm}$ ): The diameter of the lesion is recorded in millimeters. Most typical Spitz tumors measure under $10 \mathrm{~mm}$ and frequently $<5-6 \mathrm{~mm}$. 
Table 5 Histopathological criteria for atypical Spitz tumors

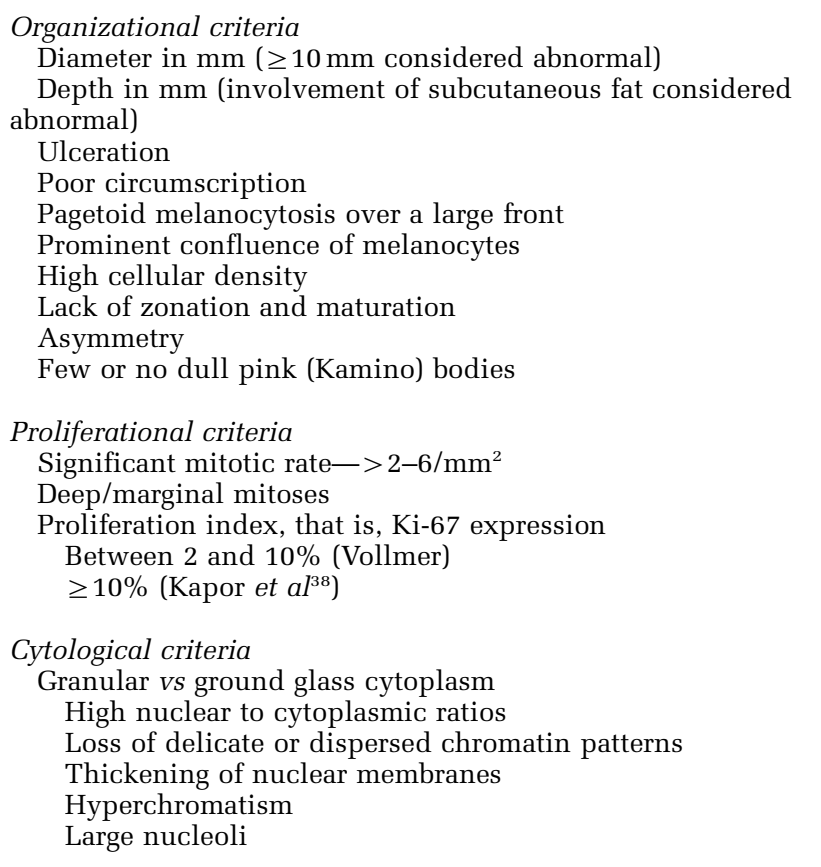

Table 6 Assessment of Spitz tumors in children and adolescents for risk for metastasis ${ }^{30}$

\begin{tabular}{lc}
\hline Parameter & Score \\
\hline Age (years) & \\
$0-10$ & 0 \\
$11-17$ & 1 \\
Diameter (mm) & \\
$0-10$ & 0 \\
$>10$ & 1 \\
Involvement of subcutaneous fat & \\
Absent & 0 \\
Present & 2 \\
Ulceration & \\
Absent & 0 \\
Present & 2 \\
Mitotic activity $\left(\mathrm{mm}^{2}\right)$ & \\
$0-5$ & 0 \\
$6-8$ & 2 \\
$>9$ & 5 \\
\hline
\end{tabular}

${ }^{\mathrm{a}}$ Total score indicates increasing risk for metastasis.

Size beyond $10 \mathrm{~mm}$ is generally considered abnormal. ${ }^{29-31}$ This is a continuous variable and there are obviously exceptions to this criterion.

Tumor thickness (measured in $\mathrm{mm}$ ): Significant (Breslow) depth and involvement of the subcutaneous fat are considered abnormal. ${ }^{30}$

Asymmetry: Increasing asymmetry is abnormal as in all melanocytic lesions. ${ }^{20}$
Ulceration: The ulceration is abnormal. ${ }^{30}$ However, since ulceration is induced by trauma in almost all instances, its significance will vary.

Poor circumscription: The most banal Spitz tumors tend to be sharply circumscribed at their peripheries whereas atypical lesions are often less well-circumscribed. ${ }^{20}$ This parameter obviously correlates with asymmetry and other organizational attributes.

Pagetoid melanocytosis: Pagetoid spread may be observed not infrequently in Spitz tumors. ${ }^{21}$ However, such pagetoid spread should be limited to the lower half of epidermis, should not extend peripherally, should be only focal, and sparsely cellular (Figure 1). More extensive pagetoid spread involving the upper half of the epidermis, a large zone of the lesion (at least one high-power field), and in a single-cell or small nested pattern is distinctly abnormal. External trauma may be a factor leading to excessive pagetoid spread in benign lesions.

Prominent confluence of melanocytes. High cellular density: These two parameters are closely correlated and are among the most important criteria for assessing melanocytic lesions. ${ }^{27}$ Unfortunately, these characteristics are subjective and therefore difficult to recognize reliably and reproducibly. These two parameters are also often closely linked to diminished or absent maturation (see below). Confluent cellular aggregates or nodules of considerable size and with crowded appearance in the dermal component, particularly replacing the dermis, and extending deep without maturation are also decidedly atypical (Figures 2 and 3). Breslow thickness obviously may capture significance of such expansile dermal nodules. One caveat is the occurrence of such nodules in Spitz tumors of young children, which may take on less importance in the latter context. ${ }^{29}$

Lack of zonation and maturation. Zonation refers to the side-to-side homogeneity often observed in typical Spitz rumors (Figure 1)..$^{20,29}$ Whereas maturation is the progressively diminished sizes of nests of melanocytes and gradual dispersion of melanocytes to smaller nests and single cells with depth. The latter phenomenon, in its most developed state, involves a nondisruptive infiltration of melanocytes among collagen bundles and involution of melanocytes to smaller cells with smaller nuclei with depth. Therefore, the nonuniformity (heterogeneity of organization) of a lesion when scanned from side to side and the continued presence of nests and fascicles of similar sizes deep indicate potentially aggressive properties (Figures 2 and 3 ).

Few or no dull pink (Kamino) bodies. Although this feature may be seen in both Spitz tumors and melanomas, the presence of clearcut aggregates of Kamino bodies may be a marker suggesting a more conventional Spitz tumor. ${ }^{32}$ 
Table 7 Immunohistochemical evaluation of Spitz tumors and atypical variants as compared to conventional nevi and malignant melanoma (from Kapur et $a l^{38}$ )

Ki-67\% p21\% Fatty acid synthase

\begin{tabular}{lccc}
\hline Banal nevi (19) & 0.53 & 0.21 & 0.21 \\
Spitz tumors (26) & 5.04 & 46.50 & 0.46 \\
Atypical Spitz tumors (10) & 10.0 & 49.25 & 1.56 \\
Malignant melan (18) & 36.83 & 27.50 & 2.28
\end{tabular}

\section{Proliferational attributes}

Mitotic rate per $\mathrm{mm}^{2}$. The mitotic rate of the dermal component is one of the most important parameters for evaluating Spitzoid lesions as increasing proliferative rate seems to correlate with likelihood of aggressive behavior or malignancy. ${ }^{30,33}$ Furthermore, this parameter is quantifiable. Mitoses observed in the deepest parts of the dermal or subcutaneous component, that is, near the deep margin, also seem to have greater significance than more superficially located mitoses. ${ }^{33}$ There are no absolute thresholds for the mitotic rate being indicative of malignancy, and the author cautions against using mitotic rate alone (or any single criterion alone) for the interpretation of a lesion as being malignant. It is important in almost all instances to have several distinctly abnormal parameters present to confirm malignancy. Every lesion must be systematically assessed on a case-by-case basis. In any case, high mitotic rates, particularly beyond six mitoses per $\mathrm{mm}^{2}$ raise considerable concern for malignancy. ${ }^{30}$ Unusual circumstances that may confound the importance of high mitotic rate include: (1) developing Spitz tumors may be in a growth phase and mitotic rate may have less significance (RL Barnhill, 2005, personal observations), (2) Spitz tumors in very young individuals may have somewhat higher mitotic rates, and (3) external trauma and significant inflammation may be factors leading to higher mitotic rates

Proliferation index, that is, Ki-67 expression. The expression of proliferation markers such as Ki-67 has taken on increasing importance for the assessment of malignant melanoma. ${ }^{34}$ As a result, the use of Ki-67 and closely related indicators may provide information beyond that of mitotic rate on the proliferation status of Spitzoid lesions. ${ }^{34-38}$ Although requiring much more study for standardization, Ki-67 expression may be useful in the risk stratification of Spitz tumors. For example, Kapor et $a l^{38}$ have recently shown that atypical Spitz tumors have a mean Ki-67 labeling index of $10 \%$ relative to $0.53 \%$ in ordinary nevi, $5.04 \%$ in conventional Spitz tumors, and $36.83 \%$ in conventional melanomas (Table 7). Vollmer's ${ }^{37}$ data suggest that a Ki-67 proliferation index less than $2 \%$ favors a conventional Spitz tumor, one greater than $10 \%$ suggests melanoma, and indices between 2 and $10 \%$ are equivocal (in the author's judgement such indices may thus suggest an atypical Spitz tumor and potentially one that is biologically indeterminate]. The qualitative loss of Ki-67 expression vs continued labeling with depth as with markers also correlates with maturation and less atypical lesion.

\section{Cytological attributes}

The assessment of cytological attributes is of great importance but remains highly subjective and lacks reproducibility. ${ }^{27}$ Cytological features suggesting a more atypical Spitzoid lesion and possibly melanoma include heterogeneity of cell type throughout the lesion, particularly in an asymmetrical or haphazard pattern; high-nuclear-to-cytoplasmic ratios; granular or 'dusty' cytoplasm vs the ground glass cytoplasm of Spitz melanocytes; absence of delicate or dispersed chromatin patterns with thickening of nuclear membranes; a large proportion of melanocytes with hyperchromatic nuclei; and the presence of large eosinophilic nucleoli.

\section{Clinical Attributes}

Age

The vast majority of Spitz tumors occur in young individuals particularly under the age of $10-20$ years. $^{2-24}$ The older the patient, especially individuals beyond the age of 20-30 years, the greater the likelihood of malignancy. As a general rule, one's threshold for diagnosing melanoma in such lesions should correlate inversely with the age of the patient, that is, a high threshold for very young individuals and a lower threshold for elderly individuals. Recently, Vollmer ${ }^{39}$ has provided guidelines for the diagnosis of Spitzoid lesions vs melanoma.

The author has nonetheless noted that Spitz tumors may occur with greater frequency in older adults than has been appreciated owing to the propensity of many pathologists to a priori interpret them as melanoma (RL Barnhill, 2005, personal observations).

\section{Other clinical attributes}

Other clinical factors such as the location of the tumor, clinical appearance, history of recent changes in a long-standing stable lesion, and family history of melanoma should be considered carefully. ${ }^{27}$ Spitz tumors commonly involve the extremities and face. The location of atypical tumors on sites less commonly involved by Spitz tumor, such as the back, is also another factor suggesting careful scrutiny of the lesion for melanoma.

\section{Special Techniques}

\section{Immunohistochemistry}

Spitzoid lesions have been evaluated with a variety of melanocytic markers. ${ }^{27,34,40-43}$ S100 protein and Mart-1 show diffuse expression throughout both 
Spitz tumors and melanoma in contrast to the characteristics diminished expression of HMB-45, tyrosinase, and other markers toward the base of Spitz tumors. ${ }^{40,41}$ In addition, there is a gradient of diminished proliferation with increasing depth of the dermal component paralleling mitotic rate and cyclin D1 expression in Spitz tumors. ${ }^{34,35,42,43}$ Spitz tumors also appear to exhibit lower rates of p53, bcl2 , and fatty acid synthase expression compared to melanoma. ${ }^{42,38}$ Fatty acid synthase may also aid in distinguishing atypical Spitz tumors from melanoma. For example, according to Kapor et $a l^{38}$ ordinary nevi show $0.21 \%$, conventional Spitz tumors $0.46 \%$, atypical Spitz tumors $1.56 \%$, and melanomas $2.28 \%$ labeling with fatty synthase. Although many of the latter markers are of interest, they require rigorous assessment with greater numbers of cases and longterm follow-up in order determine whether they have any predictive value in the evaluation of Spitzoid lesions.

\section{Comparative genomic hybridization and fluorescence} in situ hybridization

The majority of Spitz tumors studied by comparative genomic hybridization (CGH) have had no chromosomal abnormalities. ${ }^{44,45}$ On the other hand, amplification of chromosome 11p has been observed in a subset of cases and was found to correlate with Spitz tumors that were often larger in size, dermal-based, desmoplastic, had vesicular nuclei in melanocytes, and exhibited dermal infiltrating features. Further study of Spitz tumors with gains of $11 \mathrm{p}$ has shown an increase in copy number of and mutations in the HRAS gene by fluorescence in situ hybridization. The latter abnormalities do not usually occur in melanoma and consequently the authors have suggested that such findings (and the absence of chromosomal aberrations in most Spitz tumors) may aid in the distinction of Spitz tumors from melanoma. Other conclusions from the latter studies were that Spitz tumors are probably clonal proliferations, the majority of melanocytes are diploid with some large nuclei being polyploid, and Spitz tumors may be cytogenetically distinct from melanoma. ${ }^{45}$

\section{Loss of heterozygosity}

Two independent studies have recently demonstrated loss of heterozygosity on chromosome 9p with DNA polymorphic markers in two of 27 and five of five 'Spitz nevi'. ${ }^{46,47}$ The latter findings provide additional evidence for the close relationship between Spitz tumors and melanoma.

\section{Analysis of gene mutations}

Recent work has shown that a series of conventional Spitz tumors and the so-called Spitzoid melanomas (? metastasizing Spitz tumors) in prepubescent children failed to show any hotspot activating mutations in the B-raf, N-ras, or H-ras genes. ${ }^{48}$ The general absence of B-raf mutations in Spitzoid lesions contrasts with a high rate of mutation (53-
$80 \%$ ) in conventional melanomas ${ }^{49}$ and (70-90\%) in melanocytic nevi ${ }^{50}$ and suggests a different and perhaps yet to be characterized developmental pathway for Spitzoid lesions.

\section{Sentinel lymph node biopsy}

The recent application of the sentinel lymph node (SLN) biopsy to assess atypical Spitz-like tumors provides a means of obtaining more information about the biological characteristics of such lesions. ${ }^{51-53}$ Atypical Spitz-like tumors may have metastatic deposits in the peripheral sinuses or parenchyma of SLNs. ${ }^{51}$ Although in general the finding of tumor deposits in SLN is de facto evidence for melanoma, only the study of sufficient numbers of cases with long-term follow-up will provide the data to know the biological significance of such SLN deposits associated with atypical Spitz tumors, if they are inherently different from conventional melanomas, and if they potentially have better prognoses. Although large lymph node deposits demonstrating pronounced cytological atypia, prominent mitotic rates, and necrosis are almost certainly indicative of malignancy, the significance of small bland microscopic deposits is unclear and requires more detailed analysis. Almost all completion lymphadenectomies for atypical Spitz tumors with positive SLNs have failed to show any residual tumor. ${ }^{52,53}$ In addition, the latter patients have had no further disease recurrence with continued follow-up of 2-3 years. ${ }^{53}$ Atypical Spitz tumor deposits in SLNs may possibly have a different biology or significance than metastases from conventional melanoma. Many questions remain to be answered about the significance of SLN involvement by atypical Spitz tumors, particularly microscopic metastases. These findings may have relevance to the entity of the so-called 'metastasizing Spitz tumor' as some of these patients have remained disease-free with long-term follow-up of up to 15 years or more. ${ }^{29,31}$ Among the limited number of cases studied thus far by SLN biopsy, such Spitzoid tumors have not necessarily had the degree of abnormality, that is, size $>1 \mathrm{~cm}$, ulceration, involvement of subcutaneous fat, high mitotic rate, as such tumors resulting in macroscopic metastases. ${ }^{51}$

\section{Metastasizing Spitz tumor}

As already mentioned above, some melanocytic lesions classified as 'Spitz nevi' have spread to regional lymph nodes without subsequent disease progression. ${ }^{3,8,29,31,54,55}$ The concept of localized, presumably 'benign' metastases has been proposed to explain this rare phenomenon. However, some of these metastasizing melanocytic lesions, albeit resembling in many ways 'Spitz nevi', have tended to be unusually large (ie, $>1 \mathrm{~cm}$ ), often deep with involvement of subcutaneous fat, ulcerated, and to 
have high mitotic rates or showed other uncommon features (see above). ${ }^{25,29,30,31}$ An alternative explanation is that these tumors are unusual melanomas with Spitzoid features, which may or may not have a less aggressive potential for spread beyond regional lymph nodes. More definitive characterization is needed before any conclusions can be drawn about the latter group of tumors.

\section{Spitzoid melanoma}

Although it can justifiably be argued that some proportion of melanomas (perhaps large) resemble Spitz tumors and the converse, the term Spitzoid melanoma, if used at all, should be reserved for melanomas that truly have a striking morphological resemblance to Spitz tumors. ${ }^{20,27,33,56}$ The term probably best describes a rare group of tumors often developing in young individuals who are only diagnosed as melanoma in retrospect, that is, after the development of metastases and an aggressive course. Given the profound difficulty of distinguishing some Spitz tumors from melanoma, the author discourages the use of term Spitzoid melanoma since it may result in the indiscriminate labeling of a heterogeneous group of lesions including benign Spitz tumors, lesions that are biologically indeterminant, conventional melanomas, and also the rare controversial group of tumors mentioned above: 'metastasizing Spitz tumor'. The latter group of lesions includes some that have given rise to single lymph node metastases without subsequent recurrence on long-term followup. It cannot be overemphasized that as a group all of these unusual Spitz-like tumors require more detailed study as to their biological nature.

In general, there are no distinctive clinical features. ${ }^{27}$ Such Spitzoid melanomas often have abnormal clinical attributes such as size $>5-6 \mathrm{~mm}$, asymmetry, and irregular coloration suggesting an atypical nevus or melanoma. Some such lesions may suggest a Spitz tumor clinically but it must be recalled that the clinical diagnosis of a Spitz tumor is rather imprecise.

The diagnosis of a melanoma as 'Spitzoid' as mentioned above is based on the striking architectural and cytological resemblance to a Spitz tumor. ${ }^{27}$ Thus, such features potentially include any of the following: dome-shaped, plaque-like, or wedgeshaped morphology; little or no asymmetry; epidermal hyperplasia; clefting about intraepidermal nests of melanocytes; presence of dull pink or Kamino bodies; some evidence of zonation or maturation; and especially a population of enlarged epithelioid and/or spindled melanocytes with abundant opaque or 'ground glass' cytoplasms.

\section{Final analysis for diagnostic interpretation}

All histopathological, clinical, and ancillary criteria must be weighed in the final interpretation of a
Spitzoid lesion. Since we lack objective data and sufficient follow-up, the significance or weighting of the various features already mentioned has not been established. However, at present the final interpretation of a Spitzoid lesion remains almost entirely histopathological with important consideration given to clinical information. Almost all other parameters have not yet been sufficiently studied as to have any significant impact on the final interpretation. However, some indices such as the Ki-67 labelling, the gradient of expression of markers such as HMB45 (gp100) or fatty acid synthase, and CGH may provide useful information on the final deliberation about a lesion. In the future, such ancillary data may take on much greater importance.

Some may point out that such an approach seems to render many Spitz tumors atypical. In the process of evaluating Spitz tumors common sense must prevail, and one must keep in mind that one is most likely dealing with a biological continuum with many Spitz tumors at the 'benign' or 'less aggressive' end of the spectrum. There is little question that as these various parameters progressively accumulate in number and severity, the probability of an aggressive phenotype or melanoma increases. ${ }^{30}$

It is apparent that certain parameters take on more significance than others $\left({ }^{*}\right.$ represents most helpful features).$^{30}$ Potentially aggressive tumors or melanomas thus often have large size ${ }^{*}(>5-6 \mathrm{~mm}$, often $>10 \mathrm{~mm}^{*}$ ); may have significant depth*; demonstrate distinct asymmetry*; poor circumscription; heterogeneity of cellular populations*; more disordered intraepidermal proliferative patterns of melanocytes without clefting; extensive pagetoid spread; irregular epidermal alterations including thinning and effacement; significant melanocytic density and confluence*; and the lack of or zonation or diminished cellular density with depth (maturation)*. The lack of uniformity or homogeneity of cell type along comparable strata (from side to side) of the tumor cannot be overemphasized as a major criterion favoring melanoma. Similarly, the failure of a tumor to show progressive dispersion of melanocytes to smaller aggregates and particularly to single melanocytes (among apparently unaffected collagen bundles) in the deepest part of the lesion also suggests melanoma. Usually concurrent with depth is the uniform diminution of cellular and nuclear sizes and regular spacing of melanocytes in a Spitz tumor; the failure to observe the latter feature should prompt consideration of melanoma.

Cytological features favoring melanoma include alterations that are a distinct departure from what is considered acceptable for a Spitz tumor*: heterogeneity of cell type throughout the lesion, particularly in an asymmetrical or haphazard pattern; high-nuclear-to-cytoplasmic ratios; granular or 'dusty' cytoplasm vs the ground glass cytoplasm of Spitz melanocytes; absence of delicate or dispersed chromatin patterns with thickening of nuclear membranes; a large proportion of melanocytes with 
hyperchromatic nuclei; and large eosinophilic nucleoli.

As discussed above, the greater the absolute rate (per $\left.\mathrm{mm}^{2}\right)^{*}$ and number of deeply located (dermal) mitoses*, the more evidence one has favoring melanoma. Atypical mitoses and necrotic cells suggest melanoma but are not absolute.

Finally, among the clinical factors age* is of paramount importance. It is obvious that a lesion with the gross morphological features suggesting melanoma warrants special attention. A persistently changing lesion over weeks or months significantly raises concern for melanoma; whereas a lesion that is unchanged over many years argues against melanoma.

Acknowledging that this differential diagnosis is the most difficult one in melanoma pathology, there are circumstances that make it even more exasperating if not impossible. In particular, trauma and significant host response often introduce abnormal features such as asymmetry, heterogeneity, and cytological abnormality suggesting the greater likelihood of melanoma. It must be kept in mind that the nuclei in Spitz tumors are delicate and that any artefact such as tissue compression or overstaining or significant host response may introduce alterations suggesting greater cytological atypicality. In the latter circumstances, the pathologist must consider carefully all of the criteria available before rendering an interpretation.

When entertaining the possibility of melanoma, one must always consider a Spitz tumor with overlapping features of pigmented spindle cell tumor and one with phenotypic heterogeneity ('combined nevus'). Pigmented spindle cell tumors show considerable overlap with Spitz tumors and may introduce features suggesting melanoma such as greater pagetoid spread, expansile papillary dermal nests, and the absence ground glass cytoplasm. Spitz tumors with phenotypic heterogeneity ('combined nevus') may exhibit asymmetry and heterogeneity, two attributes suggesting melanoma. One must assess each component of such a lesion individually with the criteria already mentioned (Table 5), and it will usually be possible to resolve the issue.

\section{Management}

The author recommends that all Spitz tumors be fully resected in order to facilitate complete histopathological examination and also to diminish the risk of recurrence. Atypical Spitz tumors obviously require comparable excision for the same reasons but with greater clearance (up to $1 \mathrm{~cm}$ ) in order to provide even greater assurance that they are 'wholly out'. The reasons for recommending excision with margins free of the tumor are that (1) I have observed at least one Spitz tumor not completely excised that persisted (recurred) at the same site and eventuated in metastases and death, ${ }^{25}$ and (2) some persistent/ recurrent Spitz tumors may be more atypical than the original lesions and even more difficult to distinguish from melanoma. ${ }^{25,36}$ Some of the latter tumors have resulted in metastases(Barnhill et $a l^{25}$ and RL Barnhill, 2005, personal observations). It is the author's opinion that Spitz-like melanocytic tumors assigned an indeterminate biological potential require surgical margins of approximately $1 \mathrm{~cm}$ since this is considered the minimum standard of care for melanoma. The author acknowledges that there are currently no definitive data available on the issue of surgical margins for melanocytic lesions in general. Although of unproven benefit, SLN biopsy may be considered for selected lesions (generally $>1 \mathrm{~mm}$ in thickness). Patients should be carefully monitored by regular examinations for recurrence (and metastasis in the case of atypical Spitz tumors). All patients should be managed on an individual basis and efforts made to avoid both overly aggressive and suboptimal management strategies.

\section{Conclusions}

The author has attempted to portray accurately the current state of the art surrounding Spitzoid lesions, their nomenclature, and assessment of risk. Although much remains to be learned about these lesions, there is increasing evidence that they may be a type of melanocytic neoplasm distinct from conventional melanocytic nevi and malignant melanoma. Acknowledging the peculiar nature of Spitzoid lesions, the author prefers the term Spitz tumor rather than 'Spitz nevus' and argues against using the term 'Spitzoid melanoma' until more information is available to justify such a term. The author has also provided rational arguments that patients are best served by the comprehensive evaluation of Spitzoid lesions and their classification into three categories: (1) Spitz tumor without significant abnormality, (2) Spitz tumors with one or more atypical features (atypical Spitz tumor), including those judged to have indeterminate biological potential, and (3) malignant melanoma, rather than the two categories of 'Spitz nevus' and melanoma. Such an approach honestly recognizes our current lack of knowledge about many of these lsions and avoids overdiagnosis of melanoma and under recognition of potentially aggressive neoplasms. The author has outlined a protocol for this assessment of histopathological, clinical, and ancillary data and subsequent risk stratification. In conclusion, only through rigorous characterization of sufficient numbers of Spitzoid lesions and long-term follow-up of patients will we be able to have truly objective information about these lesions. Such data would allow us to finally formulate optimal guidelines for the care of patients with these lesions.

\section{Note}

Herein the terms Spitzoid or Spitz-like melanocytic lesion, tumor, or neoplasm are used generically 
and interchangeably to refer to the broad spectrum of lesions from the most conventional or typical Spitz tumor to biologically indeterminate and even aggressive or malignant lesions closely resembling Spitz tumors. Spitz tumor indicates conventional lesions ('Spitz nevi') without clearly observable abnormalities; atypical Spitz tumor refers to Spitz tumors with one or more and often several distinctly abnormal characteristics generally not present in typical Spitz tumors, and including biologically indeterminate lesions.

\section{References}

1 Darier J, Civatte A. Naevus ou naevo-carcinoma chez un nourisson. Bull Soc Franc Derm Syph 1910;21: 61-63.

2 Spitz S. Melanomas of childhood. Am J Pathol 1948;24:591-609.

3 Allen A, Spitz S. Malignant melanoma: a clinicopathological analysis of the criteria for diagnosis and prognosis. Cancer 1953;6:1-45.

4 McWorther H, Woolner L. Pigmented nevi, juvenile melanomas, and malignant melanomas in children. Cancer 1954;7:564-585.

5 Helwig E. Seminar on skin neoplasms and dermatoses. In: Proceedings of the Twentieth Seminar of the American Society of Clinical Pathologists; September 11, 1954; Am Soc Clin Pathol 1955;63-67.

6 Allen A. Juvenile melanomas of children and adults and melanocarcinomas of children. Arch Dermatol 1960;82:325-335.

7 Kernen J, Ackerman L. Spindle cell nevi and epithelioid cell nevi (so-called juvenile melanomas) in children and adults: a clinicopathological study of 27 cases. Cancer 1960;13:612-625.

8 Kopf A, Andrade R. Benign juvenile melanoma. In: Kopf A, Andrade R (eds). Yearbook of Dermatology 1965-1966. Yearbook Publishing: Chicago, IL, 1966, pp 7-52.

9 Coskey R, Mehregan A. Spindle cell nevi in adults and children. Arch Dermatol 1973;108:535-536.

10 Echevarria R, Ackerman L. Spindle and epithelioid nevi in the adult. Clinicopathologic report of 26 cases. Cancer 1967;20:175-189.

11 Paniago-Pereira C, Maize J, Ackerman A. Nevus of large spindle and/or epithelioid cells (Spitz' nevus). Arch Dermatol 1978;114:1811-1823.

12 Weedon D, Little J. Spindle and epithelioid cell nevi in children and adults. A review of 211 cases of the Spitz nevus. Cancer 1977;40:217-225.

13 Weedon D. The Spitz nevus. Clin Oncol 1984;3: 493-507.

14 Gartmann H, Ganser M. Der Spitz-Naevus. Spindelzellen und/oder Epithelienzellennaevus. Eine klinische Analyse von 652 Tumoren. Z Hautkr 1985;60:22-28.

15 Peters M, Goellner J. Spitz nevi and malignant melanomas of childhood and adolescence. Histopathology 1986;10:1289-1302.

16 Merot Y, Frenk E. Spitz nevus (large spindle and/or epithelioid cell nevus). Age-related involvement of the suprabasal epidermis. Virchows Arch A (Pathol Anat) 1989;415:97-101.

17 Elder D, Murphy G. Spindle and epithelioid cell nevus (Spitz nevus). Atlas of tumor pathology. In: Elder D,
Murphy G (eds). Melanocytic Tumors of the Skin. Armed Forces Institute of Pathology: Washington, 1990, pp 40-57.

18 Mooi W, Krausz T. Spitz naevus, desmoplastic Spitz naevus and pigmented spindle cell naevus. In: Mooi W, Krausz T (eds). Biopsy: Pathology of Melanocytic Disorders. Chapman \& Hall: London, 1992, pp 156-185.

19 Binder S, Asnog C, Paul E, et al. The histology and differential diagnosis of Spitz nevus. Semin Diagn Pathol 1993;10:36-46.

20 Barnhill RL, Piepkorn M, Busam KJ. The Pathology of Melanocytic Nevi and Malignant Melanoma, 2nd edn. Springer-Verlag: New York, 2004.

21 Busam KJ, Barnhill RL. Pagetoid Spitz nevus. Intraepidermal Spitz tumor with prominent pagetoid spread. Am J Surg Pathol 1995;19:1061-1067.

22 Piepkorn M. On the nature of histologic observations: the case of the Spitz nevus. J Am Acad Dermatol 1995;32:248-254.

23 Cramer SF. The melanocyte differentiation pathway in Spitz nevi. Am J Dermatopathol 1998;20:555-570.

24 Mooi WJ. Spitz nevus and its histologic simulators. Adv Anat Pathol 2002;9:209-221.

25 Barnhill RL, Argenyi ZB, From L, et al. Atypical Spitz nevi/tumors: lack of consensus for diagnosis, discrimination from melanoma, and prediction of outcome. Hum Pathol 1999;30:513-520.

26 Reed R, Ichinose H, Clark W, et al. Common and uncommon melanocytic nevi and borderline melanomas. Semin Oncol 1975;2:119-147.

27 Barnhill RL, Piepkorn M, Busam KJ. The Pathology of Melanocytic Nevi and Malignant Melanoma, 2nd edn. Springer-Verlag: New York, 2004.

28 Mones JM, Ackerman AB. Atypical. Spitz's nevus, 'malignant' Spitz's nevus, and 'metastasizing' Spitz's nevus: a critique in historical perspective of three concepts flawed fatally. Am J Dermatopathol 2004;26: 310-333.

29 Barnhill RL, Flotte T, Fleischli M, et al. Childhood melanoma and atypical Spitz-tumors. Cancer 1995;76: 1833-1845.

30 Spatz A, Calonje E, Handfield-Jones S, et al. Spitz tumors in children: a grading system for risk stratification. Arch Dermatol 1999;135:282-285.

31 Smith K, Skelton H, Lupton G, et al. Spindle cell and epithelioid cell nevi with atypia and metastasis (malignant Spitz nevus). Am J Surg Pathol 1989;13: 931-939.

32 Kamino H, Misheloff E, Ackerman A, et al. Eosinophilic globules in Spitz's nevi. New findings and a diagnostic sign. Am J Surg Pathol 1979;1:319-324.

33 Walsh N, Crotty K, Palmer A, et al. Spitz nevus versus spitzoid malignant melanoma: an evaluation of the current distinguishing histopathologic criteria. Hum Pathol 1998;29:1105-1112.

$34 \mathrm{Li}$ LX, Crotty KA, McCarthy SW, et al. A zonal comparison of MIB1-Ki67 immunoreactivity in benign and malignant melanocytic lesions. Am J Dermatopathol 2000;22:489-495.

35 Bergman R, Malkin L, Sabo E, et al. MIB-1 monoclonal antibody to determine proliferative activity of Ki-67 antigen as an adjunct to histopathologic differential diagnosis of Spitz nevi. J Am Acad Dermatol 2001;44:500-504.

36 Harvell JD, Bastian BC, LeBoit PE. Persistent (recurrent) Spitz Nevi: A histopathologic, immunohisto- 
chemical, and molecular pathologic study of 22 cases. Am J Surg Pathol 2002;26:654-661.

37 Vollmer RT. Use of Bayes rule and MIB-1 proliferation index to discriminate Spitz nevus from malignant melanoma. Am J Clin Pathol 2004;122:499-505.

38 Kapor P, Selim MA, Roy LC, et al. Spitz nevi and atypical Spitz nevi/tumors: a histologic and immunohistochemical analysis. Mod Pathol 2005;18:197-204.

39 Vollmer RT. Patient age in Spitz nevus and malignant melanoma: implication of Bayes rule for differential diagnosis. Am J Clin Pathol 2004;121:872-877.

40 Rode J, Williams R, Jarvis L, et al. S-100 protein, neuron-specific enolase, and nuclear DNA content in Spitz nevus. J Pathol 1990;161:41-45.

41 Bergman R, Dromi R, Trau H, et al. The pattern of HMB-45 antibody staining in compound Spitz nevi. Am J Dermatopathol 1995;17:542-546.

42 Kanter-Lewensohn L, Hedblad MA, Wejde J, et al. Immunohistochemical markers for distinguishing Spitz nevi from malignant melanomas. Mod Pathol 1997;10:917-920.

43 Ewanowich C, Brynes R K, Medeiros J, et al. Cyclin D1 expression in dysplastic nevi. Arch Pathol Lab Med 2001;125:208-210.

44 Bastian BC, Wesselman U, Pinkel D. LeBoit Molecular cytogenetic analysis of Spitz nevis shows clear differences to melanoma. J Invest Dermatol 1999;113: 1065-1069.

45 Bastian BC, LeBoit PE, Pinkel D. Mutations and copy number increase of HRAS in Spitz nevi with distinctive histopathologic features. Am J Pathol 2000;157:967-972.
46 Healy E, Belgaid C, Takata M, et al. Allelotypes of primary cutaneous melanoma and benign melanocytic nevi. Cancer Res 1996;56:589-593.

47 Bogdan I, Burg G, Boni R. Spitz nevi display allelic deletions. Arch Dermatol 2001;137:1417-1420.

48 Gill M, Cohen J, Renwick N, et al. Genetic similarities between Spitz nevus and Spitzoid melanoma in children. Cancer 2004;101:2636-2640.

49 Davies H, Bignell GH, Cox C, et al. Mutations of the BRAF gene in human cancer. Nature 2002;417:949-954.

50 Pollock PM, Harper UL, Hansen KS, et al. High frequency of BRAF mutations in nevi. Nat Genet 2003; 33:19-20.

51 Lohman CM, Coit DG, Brady MS, et al. Sentinel lymph node biopsy in paitents with diagnositcally controversial spitzoid melanocytic tumors. Am J Surg Pathol 2002;26:47-55.

$52 \mathrm{Su}$ LD, Fullen DR, Sondak VK, et al. Sentinel lymph node biopsy for patients with problematic spitzoid melanocytic lesions: a report on 18 patients. Cancer 2003;97:499-507.

53 Roaten JB, Partrick DA, Pearlman N, et al. Sentinel lymph node biopsy for melanoma and other melanocytic tumors in adolescents. J Pediatr Surg 2005;40: 232-235.

54 Reed R. Malignant Spitz nevus Case presented at the Clinicopathology Conference, American Academy of Pathology Meeting; New York, December 1980.

55 Delacretaz J. Melanoma juvenile (melanome de Spitz) a evolution maligne. Dermatologica 1969;139:79-83.

56 Okun MR. Melanoma resembling spindle and epithelioid cell nevus. Arch Dermatol 1979;115:1416-1420. 